$\begin{aligned} \text { Lekn }-\cdots \quad \cdots & =53 \\ & \text { su } 3402\end{aligned}$

CERN-TH.7115/93

BI-TP $93 / 66$

\title{
THE QUANTUM-MECHANICAL ORIGIN OF NUCLEAR SHADOWING
}

\author{
D. Kharzeev ${ }^{1,2}$ and H. Satz ${ }^{2,3}$
}

\begin{abstract}
:
We show that the shadowing effects observed in hadron and photon interactions with nuclei are due to quantum-mechanical coherence and interference. As such, they differ in different reactions and cannot be treated as nuclear modifications of parton structure functions. We apply this to hadron- and photon-induced charmonium production on nuclear targets, obtaining shadowing in the first case and anti-shadowing in the latter.
\end{abstract}

1) Physics Department, Moscow State University, 119899 Moscow, Russia

2) Theory Division, CERN, CH-1211 Geneva, Switzerland

3) Fakultät für Physik, Universität Bielefeld, D-33501 Bielefeld, Germany

CERN-TH.7115/93

BI-TP 93/66

December 1993 
Hard partonic interactions of hadrons fall into a regime of strong interaction physics which in principle is fully accounted for by perturbative QCD. Hence nuclear modifications of such interactions are of particular interest: they indicate where long-range hadronic features begin to change the short-range world of the perturbative approach. Conversely, they constitute a tool to probe the non-perturbative large-scale nature of nuclei and nuclear interactions by means of processes understood in terms of perturbative short-range interactions.

We know today quite a number of what seem to be nuclear modifications of perturbative phenomena. The EMC effect [1] shows that there are fewer hard partons in nuclei than there are in hadrons, while nuclear shadowing [2] indicates that there are fewer soft partons in nuclei than in hadrons. For quarks we obtain this information by scattering of (real or virtual) photons on nucleons and on nuclei; for gluons, we compare hadron-induced quarkonium production on nuclei to that on nucleons. To satisfy sum rules for parton distributions, shadowing and EMC effect regions in the Bjorken variable $x$ of the nucleus must be separated by a certain range of anti-shadowing, so that we find nuclear enhancement as well as nuclear suppression. Moreover, these effects depend on the atomic mass number of the nucleus, and they change with changing kinematics: thus the quarkonium suppression on nuclear targets increases with the longitudinal momentum of the produced resonances, with the incident energy, and as we go from bottonium to charmonium states $[3,4]$. This provides us with a variety of ways to check our understanding of the phenomena.

The present paper has two closely related aims. First, we want to show that the behaviour of the gluon structure functions through the shadowing and anti-shadowing regime is not due to any nuclear modifications of parton distributions, but simply to quantum-mechanical coherence effects. Secondly, and to prove the claim just made, we will show that the correct quantum-mechanical description of the coherent interaction of charges in a medium [5] indeed describes all results of hadron-nucleus and photonnucleus interactions in the shadowing/anti-shadowing region. Moreover, this account also results in a number of consequences for other aspects of hard interactions on nuclei. Its main prediction is the absence of a certain unversality inherent in the parton model. The photoproduction and the hadroproduction of quarkonium states on nuclei are in a parton picture very similar processes; in contrast, our understanding of shadowing and anti-shadowing as quantum-mechanical coherence effects predicts them to be quite different and not describable in terms of nuclear modifications to structure functions [6]

We will concentrate mainly on gluon structure functions, which can be extracted from data on quarkonium production; related questions involving quark structure functions will be taken up briefly towards the end of the paper. Moreover, we begin with photoproduction of quarkonium, since here strong interactions enter at one vertex only. In perturbative QCD, quarkonium photoproduction on protons, $\gamma+p \rightarrow Q \bar{Q}+X$, is treated as a photon-gluon fusion process [7], and the resulting cross section becomes the convolution of the elementary photon-gluon fusion cross section $\sigma_{\gamma g} \sim|M(\gamma g \rightarrow(Q \bar{Q}) g)|^{2}$ with the gluon density $G(x)$ in the nucleon,

$$
\sigma(\gamma p \rightarrow Q \bar{Q}) \sim \int d x G(x) \sigma_{\gamma g}
$$


For $Q \bar{Q}$ production on nuclear targets, a similar convolution form is usually assumed,

$$
\sigma(\gamma A \rightarrow Q \bar{Q}) \sim \int d x G_{A}(x) \sigma_{\gamma g}
$$

and the difference between the normalized nuclear cross section and the one on hydrogen is then attributed to modifications of the gluon density $G_{A}(x)$ inside the nucleus.

To see when the nuclear form (2) is applicable, we have to consider in some detail the space-time picture of the process. First we note that through unitarity the cross section for the virtual photoproduction of a $Q \bar{Q}$ state is proportional to the imaginary part of the forward scattering amplitude of a $Q \bar{Q}$ pair off the nucleus (see Fig.1):

$$
\operatorname{Im} F\left(s, q^{2}\right)=e^{2} \int d^{4} x_{1} d^{4} x_{2} e^{i q x}<A\left|T\left\{V\left(-x_{1}\right) V\left(x_{2}\right)\right\}\right| A>
$$

where $q$ is the four momentum of the pair, $x=\left(x_{1}-x_{2}\right)$, and $s$ the total c.m.s. energy. For a physical $Q \bar{Q}$ state we have $q^{2} \simeq 4 M_{Q}^{2}$, in contrast to $q^{2}=0$ for real photons and $q^{2}<0$ for deep-inelastic scattering. In the calculation of the photoproduction cross-section, the $Q \bar{Q}$ pair in the scattering amplitude (3) is therefore highly virtual.

If we assume that there are no dynamical internucleon correlations inside the nucleus, the nuclear matrix element can be factorized into individual nucleon contributions,

$$
<A\left|T\left\{V\left(-x_{1}\right) V\left(x_{2}\right)\right\}\right| A>=A<N\left|T\left\{V\left(-x_{1}\right) V\left(x_{2}\right)\right\}\right| N>
$$

and this leads to a nuclear cross section $\sigma_{A}=A \sigma_{N}$. A break-down of this relation would thus seem to indicate the presence of internucleonic interactions which modify the parton distributions inside the nucleus and spoil the factorization.

However, factorization can break down also in the absence of any nuclear modification of parton distributions. In particular, it fails even for dynamically uncorrelated nucleons, if the longitudinal distance over which the virtual $Q \bar{Q}$ can propagate inside the nucleus is larger than the characteristic internucleonic distance. To obtain an estimate of this "coherence length" of virtual behaviour, we consider the momentum transfer $\Delta q$ necessary to bring the virtual $Q \bar{Q}$ on shell in the field of the nucleus [8]. The energy $p_{0}$ of the $Q \bar{Q}$ pair is

$$
p_{0}=\sqrt{s_{Q}+p_{Q}^{2}}
$$

with $p_{Q}$ for the three-momentum of the $Q \bar{Q}$ system and $s_{Q} \simeq 4 M_{Q}^{2}$ for its invariant mass. In the target rest frame we have $q_{0} \simeq p_{0}$; hence for real photons, with $q^{2}=0$ and $|\mathbf{q}|=q_{0}$, the momentum transfer becomes

$$
\Delta q=\sqrt{p_{0}^{2}-s_{Q}}-q_{0} \simeq\left(s_{Q} / 2 q_{0}\right)
$$

From the uncertainty relation the coherence length $z_{c}$ is thus given by

$$
z_{c} \simeq\left(\frac{1}{\Delta q}\right) \simeq\left(\frac{2 q_{0}}{s_{Q}}\right)
$$


For sufficiently energetic photons, the virtual $Q \bar{Q}$ will therefore interact over a large distance, which increases with increasing photon energy $q_{0}$ [9]. Hence we have to check if the convolution formula (2) still correct when $z_{\mathrm{c}}$ becomes larger than the internucleonic distance in the nucleus. We shall here concentrate on real photons, to be compared to onshell gluons, and comment on differences for deep-inelastic scattering where appropriate.

The quantum-mechanical effects for the corresponding electromagnetic problem were already studied forty years ago $[5,10]$ and are readily adapted to our case. Consider first the case when the coherence length $z_{c}$ is smaller than the average internucleonic distance, $z_{c}<d$, with $d \simeq n_{o}^{-1 / 3}$, where $n_{o}$ is the average nuclear density. In this case the amplitudes of $Q \bar{Q}$ production on different nucleons do not interfere, and in the absence of nuclear effects the nuclear cross section is just $\sigma(\gamma A \rightarrow Q \bar{Q})=A \sigma(\gamma N \rightarrow Q \bar{Q})$. This situtation undergoes a fundamental change as $z_{c}$ increases. The mean free path $\lambda$ of the virtual $Q \bar{Q}$ fluctuation in nuclear matter now enters as a new scale,

$$
\lambda=\left(\sigma n_{o}\right)^{-1},
$$

where $\sigma$ is the cross section for the interaction of the virtual $Q \bar{Q}$ system on nucleons, and a very interesting quantum-mechanical phenomenon appears in the regime $d<z_{c}<\lambda$. To illustrate its origin, let us imagine a hypothetical crystalline nucleus, in which the nucleons are spaced at equal separation distance $d$ along some axis. If the $Q \bar{Q}$ quantum enters the crystal along this axis, then the $N \simeq z_{c} / d$ elementary amplitudes $M$ within the coherence length $z_{c}$ add coherently, and the resulting cross section $\sigma_{\text {coh }} \sim|N M|^{2}$ is $N$ times larger than the incoherent sum $\sigma_{\text {inc }} \sim N|M|^{2}$. The maximum value of coherently contributing amplitudes is $N \simeq \lambda / d$, with $z_{c} \leq \lambda \leq R_{A}$. In this range, the nuclear cross-section thus is larger than the incoherent sum of nucleon cross-sections by a factor of $\lambda / d$ :

$$
\sigma(\gamma A \rightarrow Q \bar{Q}) \simeq\left(\frac{\lambda}{d}\right) A \sigma(\gamma N \rightarrow Q \bar{Q})
$$

This effective enhancement is completely analogous to the enhancement of electron bremsstrahlung radiation which occurs when a relativistic electron enters the crystal along its axis and the coherence length $z_{c}$ of the radiation exceeds the interatomic distance $d[8]$.

A real nucleus of course differs from a crystal, since the nucleons are located randomly inside the nuclear volume. It is therefore very unlikely to find coherence of more than two elementary amplitudes. Even this requires that the quantum passes through the partonic core of two nucleons within the coherence length. The probability of this event per length $d$ can be roughly estimated as $\left(r_{c} / d\right)^{2}$, where $r_{c} \simeq 0.6-0.8 \mathrm{fm}$ is the radius of the partonic core of the nucleon. The total probability along the coherence length $z_{c}$ is then $\left(r_{c} / d\right)^{2}\left(z_{c} / d\right)$, and since the regime holds until $z_{c} \simeq \lambda$, we conclude that quantum coherence can enhance the nuclear cross section by a maximum amount of $\left[1+\left(r_{c}^{2} / d^{2}\right)(\lambda / d)\right]$. For $\lambda \leq R_{A}$, this leads to an anti-shadowing which essentially does not depend on the atomic number of the nucleus. On the other hand, if $\lambda \geq R_{A}$, as in the case of a heavy colour-singlet $Q \bar{Q}$ pair, then the factor $\lambda / d$ is replaced by $R_{A} / d$. The nuclear cross section now becomes enhanced by a factor $\left[1+\left(r_{c} / d\right)^{2}\left(R_{A} / d\right)\right]$, so that the amount of anti-shadowing now depends on $A$ through $R_{A}$. 
When $z_{\mathrm{c}}$ becomes larger than the mean free path length $\lambda$, the coherence is broken by multiple scattering, and for sufficiently large systems (allowing $\lambda<<z_{c}<<R_{A}$ ), the contributions from different scatterings interfere destructively; this is the LandauPomeranchuk effect [5]. The loss of coherence and the interference thus lead to a suppression of the $Q \bar{Q}$ production probability. The $Q \bar{Q}$ fluctuation needs a length $z_{c} \simeq 2 q_{0} / s_{Q}$ to get on-shell, as is obtained in eq. (6) and the subsequent discussion. Multiple scattering reduces the effective range of coherent interaction from $z_{c}$ to the mean free path length $\lambda$, and when $z_{c}>>\lambda$, this results in suppression by a factor $\lambda / z_{c}$. Antishadowing is thus followed by shadowing, as $z_{c}$ increases.

In principle, a detailed study of the correlator in eq. (3) is required to determine the amount of shadowing. However, a simple estimate can easily be obtained [11]. The nuclear cross section is just the product of the probability that the photon hits the nucleus $\left(\sim \pi R_{A}^{2}\right)$, the probability that it develops a $Q \bar{Q}$ fluctuation inside the nucleus $\left(\sim R_{A} e^{2} / z_{c}\right)$, and the quantum-mechanical suppression factor $\left(\lambda / z_{c}\right)$ obtained above. Hence we get

$$
\sigma(\gamma A \rightarrow Q \bar{Q}) \sim \pi R_{A}^{2} \frac{R_{A} e^{2}}{z_{c}} \frac{\lambda}{z_{c}},
$$

with $e^{2}=1 / 137$. To see what this means, we make a similar estimate for the incoherent case $z_{c}<d<\lambda$. The factors here are the same except the last one, which now becomes the probability $z_{c} / \lambda$ that the short-lived $Q \bar{Q}$ fluctuation interacts with a nucleon inside the nucleus over the distance $z_{c}$ :

$$
\sigma(\gamma A \rightarrow Q \bar{Q}) \sim \pi R_{A}^{2} \frac{R_{A} e^{2}}{z_{c}} \frac{z_{c}}{\lambda} \sim A \sigma(\gamma N \rightarrow Q \bar{Q})
$$

From eqs. (10) and (11) we conclude that for $z_{\mathrm{c}}>\lambda$, the nuclear cross section becomes

$$
\sigma(\gamma A \rightarrow Q \bar{Q}) \simeq\left(\frac{\lambda}{z_{c}}\right)^{2} A \sigma(\gamma N \rightarrow Q \bar{Q})
$$

and thus differs from the incoherent sum by a factor $\left(\lambda / z_{c}\right)^{2}$. Note that in the shadowing range thus determined, we have assumed $\lambda<<z_{c}<<R_{A}$; when the value of $z_{c}$ approaches $R_{A}$, finite size modifications of the Landau-Pomeranchuk effect must be taken into account.

Finally, at very high energies, when $z_{c}$ exceeds the nuclear radius, the $Q \bar{Q}$ fluctuations will remain virtual throughout the whole nucleus. They will therefore interact with the nucleus mainly through intermediate vector meson fluctuations, with a typical hadronic cross section $\sim \pi R_{A}^{2}$, multiplied by $e^{2}$. We thus reach the vector meson dominance regime [12].

We can summarize the results obtained so far by saying that in the photoproduction of $Q \bar{Q}$ pairs there can be four regimes, determined by the size of the coherence length relative to the nuclear parameters $d, \lambda$ and $R_{A}$, with $d<\lambda<R_{A}$.

- Incoherence: $z_{c} \leq d$.

- Antishadowing: $d \leq z_{\mathrm{c}} \leq \lambda$.

- Shadowing: $\lambda \leq z_{c} \leq R_{A}$. 
- Vector Meson Dominance: $R_{A} \leq z_{c}$.

Let us now see how these regimes appear in terms of an an effective "modification" of the nuclear gluon structure functions.

We first define the coherence length $z_{c}$ in terms of the usual kinematical variables of the parton model. The fraction $x_{t}$ of the target nucleon momentum carried by the gluon in $\gamma+g \rightarrow Q \bar{Q}$ is given by

$$
x_{t}=\frac{s_{Q}}{s},
$$

where $s_{Q}$ is the invariant mass of the $Q \bar{Q}$ pair. The photon-nucleon c.m.s. energy $s$ becomes in the lab $s \simeq 2 m q_{0}$, where $q_{0}$ is the lab momentum of the photon and $m$ the nucleon mass. The coherence length $z_{c} \simeq 2 q_{0} / s_{Q}$ therefore is simply obtained as

$$
z_{c}^{-1}=m x_{t}
$$

and all our previous considerations can be equivalently reformulated in terms of $x_{t}=$ $\left(m z_{c}\right)^{-1}$. The basic quantity to study is the ratio

$$
R_{A / p}\left(x_{t}\right)=\frac{\sigma(\gamma A \rightarrow Q \bar{Q})}{A \sigma(\gamma p \rightarrow Q \bar{Q})}
$$

which in the parton model is considered as the ratio of nuclear to proton structure functions.

Let us now identify the above regimes in terms of $R_{A / p}$ and the Bjorken variable $x_{t}$; we just reverse the order.

- Vector Meson Dominance: $x_{t} \leq\left(m R_{A}\right)^{-1}$. In this region, $R_{A / p}$ does not depend on $x_{t}$ and becomes

$$
R_{A / p} \simeq \frac{\lambda}{R_{A}}=\left(\frac{\lambda}{r_{0}}\right) A^{-1 / 3}
$$

with $R_{A}=r_{0} A^{1 / 3}, r_{0} \simeq 1.1 \mathrm{fm}$.

- Shadowing: $\left(m R_{A}\right)^{-1} \leq x_{t} \leq(m \lambda)^{-1}$. According to eq. (11), $R_{A / p}$ here increases with $x_{t}$ roughly as

$$
R_{A / p} \simeq(m \lambda)^{2} x_{t}^{2}
$$

and does not depend on $A$.

- Antishadowing: $(m \lambda)^{-1} \leq x_{t} \leq(m d)^{-1}$. Here coherence effects increase $R_{A / p}$ to give

$$
R_{A / p} \simeq\left(1+\frac{r_{c}^{2}}{d^{2}} \frac{\lambda}{d}\right)
$$

when $\lambda \leq R_{A}$ holds. For $\lambda>R_{A}$ and $\left(m R_{A}\right)^{-1} \leq x_{t} \leq(m d)^{-1}$,

$$
R_{A / p} \simeq\left(1+\frac{r_{c}^{2}}{d^{2}} \frac{r_{0}}{d} A^{1 / 3}\right)
$$

since the nuclear radius now takes the place of the mean free path length in the preceding relation. 
- Incoherence: For $x_{t} \geq(m d)^{-1} \simeq 0.12-0.15$, all of the quantum-mechanical effects discussed above should disappear. In this region, any difference between the extracted nuclear and proton gluon structure functions can therefore be attributed to a true modification of the gluon distribution owing to dynamical nuclear effects (including Fermi motion).

The resulting behaviour of the ratio $R_{A / p}$ as a function of $x_{t}$ is illustrated in Fig. 2. It is evident that our quantum-mechanical treatment correctly reproduces, on a qualitative level, the familiar pattern of shadowing and anti-shadowing. It does so without any nuclear modification of parton distributions, just by correctly taking into account the relevant quantum-mechanical interference effects.

A striking consequence of the approach developed here is that it predicts the extracted effective nuclear structure functions to be different when tested by different processes. To show this, we shall consider two cases in some detail: the nuclear gluon structure function as extracted from deep-inelastic scattering or photoproduction of charmonium vs. that obtained from hadroproduction, and the quark structure function as obtained from deep-inelastic scattering vs. Drell-Yan dilepton production.

In the photoproduction of quarkonium, the photon turns into a small colour neutral $Q \bar{Q}$ pair, which has a large mean free path in the nuclear medium (colour transparency), with $\lambda_{0}=\left(n \sigma_{0}\right)^{-1} \geq R_{A}$. In hadroproduction, on the other hand, the gluon turns into a virtual colour octet $Q \bar{Q}$ pair. The small size of the pair is now no longer a reason for weak interactions, since even pointlike colour charges should undergo strong interactions in a nuclear medium. We thus expect that the mean free path of the colour octet $Q \bar{Q}$ state in the nucleus is not much larger than the internucleonic distance: $\lambda_{8} \sim d$. This difference in mean free paths implies a very different behaviour for $R_{A / p}$ in photo- and hadroproduction.

For photoproduction, the crucial consequence is the absence of the shadowing regime. The mean free path of the colour singlet $Q \bar{Q}$ pair, $\lambda_{0} \simeq\left(n \sigma_{0}\right)^{-1}$, is larger than the nuclear radius $R_{A}$ for any reasonable value of the cross section ( $\left.\sigma_{0} \lesssim 10 \mathrm{mb}\right)$, so that there is no region with $\left(m R_{A}\right)^{-1}<x_{t}<(m \lambda)^{-1}$. However, in the region of $\left(m R_{A}\right)^{-1} \leq x_{t} \leq(m d)^{-1}$, in which formula (18) is applicable, we predict substantial anti-shadowing. Such an enhancement was indeed observed by the NMC Collaboration [13] in deep-inelastic muon scattering * on tin and carbon, and recently confirmed on various heavy targets [14]. The NMC group studied inelastic $J / \psi$ production by virtual photons in the range $x_{t} \simeq 0.05-0.15$ and found $R_{S n / C}^{\gamma}=1.13 \pm 0.08$; they interpreted this as "an enhancement of the gluon distribution of tin with respect to carbon" [13]. However, as we have shown, a similar pattern for $R_{A / p}^{\gamma}$ arises as a natural consequence of quantum mechanics. From eq. (18) we get

$$
R_{S n / C}^{\gamma}=R_{S n / p}^{\gamma} / R_{C / p}^{\gamma} \simeq 1.1, \quad 0.04 \leq x_{t} \leq 0.12-0.15,
$$

* For deep-inelastic scattering, our estimate (7) for the correlation length must be slightly modified, since we now no longer have $q^{2}=0$. For $q_{0}^{2}>Q^{2} \equiv-q^{2}>0$, we get $z_{c}^{-1} \simeq m x_{t}\left[1+\left(Q^{2} / 4 M_{Q}^{2}\right)\right]$ instead of eq. (14). In the quoted experiment we have on the average $Q^{2} / 4 M_{Q}^{2} \simeq 1$, so that there $z^{-1} \simeq 2 m x_{t}$. This shortening of the correlation length, however, does not effect our argument. 
in agreement with the experimental value.

In the case of hadroproduction, $J / \psi$ data $[3,4]$ at $0 \leq x_{F} \leq 0.5$ in high energy hadron-nucleus collisions scale as a function of $x_{t}$ and can therefore be used to extract the ratio of nuclear and proton gluon structure functions in the interval of $0.01 \leq x_{t} \leq 0.15$ [15]. Although this is the same region as considered above for virtual photoproduction, the resulting ratio $R_{A / p}^{h}$ now is smaller than unity everywhere and decreases towards smaller values of $x_{t}$.

This becomes a discrepancy only if we try to interpret the data in terms of nuclear modifications of parton distributions; in our approach, the difference in behaviour is predicted. In hadroproduction, the mean free path length of the colour octet $Q \bar{Q}$ fluctuation is not much larger than the internucleonic distance, $\lambda_{8} \sim d$. Therefore here the anti-shadowing regime of eq. (18) is never attained. Instead, we have shadowing; it starts at $x_{t} \simeq(m d)^{-1} \simeq 0.12-0.15$, becomes more substantial for decreasing $x_{t}$, in accord with eq. (17), and then saturates for $x_{t} \leq\left(m r_{0}\right)^{-1} A^{-1 / 3}$ at the value $\left(\lambda_{8} / r_{0}\right) A^{-1 / 3}$ (see eq. (16)). For a nucleus such as tungsten this implies

$$
R_{W / p}^{h} \simeq 0.4, \quad x_{t} \leq 0.03 .
$$

Such a pattern is in fact seen in the data [10].* Again, we note that our explanation does not involve any true nuclear modification of the gluon density, neither for photonor for hadroproduction. The emerging picture is nevertheless highly non-trivial, since it implies that at high energies we enter the regime of coherent colour interactions extended over the nuclear volume.

Our considerations also allow us to understand immediately why deep-inelastic leptoproduction involving light quarks leads to shadowing, while that of heavy quarks results in anti-shadowing. In both cases we have a virtual photon turning into a colourless quark-antiquark state; the light pair, however, eventually becomes quite large and hence its interaction is not suppressed by colour transparency, as is the case for the heavy pair. The mean free path for the light pair is thus much shorter, and as a result the shadowing region $z_{c}<\lambda$ can be reached.

The Drell-Yan process on nuclei (see Fig. 3) provides another interesting test for our considerations. In the parton model, the Drell-Yan production cross-section is calculated using the quark and antiquark structure functions extracted from deep-inelastic lepton scattering data on nuclei. These structure functions show substantial shadowing at small $x_{t}$ as well as some anti-shadowing at $x_{t} \sim 0.1$. Since the cross-section for DrellYan production at high energy and/or large Feynman $x_{F}$ involves structure functions at very small $x_{t}$, the parton model predicts the presence of pronounced nuclear effects.

In our approach, this is not the case. We claim that the reason for shadowing and anti-shadowing on nuclear targets is the finite time needed to form a composite system, since in this time the system can undergo coherent interactions inside the nucleus. The pointlike virtual photon produced by quark-antiquark annihilation in the

* At still smaller $x_{t}$, an energy loss due to colour interactions of the $Q \bar{Q}$ with the medium is likely to cause an additional suppression [16]. 
basic Drell-Yan process (Fig. 3a) is formed instantaneously, just as a positron annihilates instantaneously in a crystal. Hence there can be no coherence effects, and in particular no shadowing or anti-shadowing. This argument is modified by higher order corrections to the basic Drell-Yan diagram. Coherence effects can occur through hadronic quark-antiquark fluctuations of the virtual photon mediating the process (Fig. $3 \mathrm{~b}$ ); these are, however, suppressed by an additional power of $e^{2}=1 / 137$ and should thus be rather weak. Similarly, the initial quark-antiquark fusion is no longer pointlike and hence instantaneous when higher order vertex corrections are taken into account (Fig. 3c). They induce a finite space-time duration of the process and can in principle cause coherence effects. Nevertheless, the situation here is very different from deepinelastic scattering, where the shadowing is of nonperturbative nature and arises from the interaction of large-size $q \bar{q}$ pairs with the nuclear medium. We predict therefore that, up to perturbative $\alpha_{s}^{2}$ corrections, Drell-Yan production on nuclei will show no shadowing or anti-shadowing effects in the region $x_{t} \leq 0.15$. This is in striking contradiction to the parton model conclusions, which predict essentially the same shadowing as observed in deep-inelastic scattering.

From experiments on proton-nucleus collisions at $800 \mathrm{GeV}$ one indeed concludes that deep-inelastic lepton scattering data exhibit a more pronounced shadowing at small $x_{t}$ than Drell-Yan production [17]. Moreover, nuclear Drell-Yan cross sections show no clear signature of anti-shadowing at larger $x_{t}$, again in contrast to the deep-inelastic data. Nevertheless, one would need high precision measurements extending to smaller values of $x_{t}$ in order to reach a definite conclusion.

Finally we note two consequences of our considerations which are of more theoretical nature. We obtain, at least on a qualitative level, the pattern of nuclear shadowing and anti-shadowing observed in different processes without assuming any true modification of the parton distributions in nuclei. Hence, given the parton distributions in nucleons, it should therefore be possible to calculate also quantitatively the shadowing and antishadowing effects in interactions with nuclear targets. And furthermore, if a QCD calculation of the $x_{t}$ dependence of parton distributions in nuclear matter "from first principles" should become possible, it will very likely not find any modifications of structure functions in the shadowing and anti-shadowing regime $\left(x_{t} \leq 0.15\right)$.

To summarize, we have considered the space-time picture of nuclear processes induced by photons and hadrons. At high energies, these processes occur over large longitudinal distances throughout the nuclear volume. Hence quantum-mechanical effects arising from the interference of the interaction-amplitudes from different nucleons must be taken into account. Such effects are shown to lead to anti-shadowing and shadowing regions for interactions with nuclear targets. Simple estimates show that this picture is quite consistent with present experimental results without any modification of the parton distribution in nuclei. Our considerations moreover imply that nucleus-nucleus collisions at sufficiently high energies will also result in coherent colour interactions. Therefore we have little reason to expect that a simple-minded multiple scattering picture will be applicable there, and much more work is needed to understand the QCD of high energy nuclear collisions. 


\section{References:}

1) J. J. Aubert et al., Phys. Lett. B213 (1983) 275.

2) V. Heynen et al., Phys. Lett. B34 (1971) 651;

J. Bailey et al., Nucl. Phys. B151 (1979) 367.

3) J. Badier et al., Z. Phys. C20 (1983) 101.

4) D. M. Alde et al., Phys. Rev. Lett. 66 (1991) 133 and 2285.

5) L. D. Landau and I. Ya. Pomeranchuk, Dokl. Akad. Nauk SSSR 92 (1953) 535 and 735 .

6) N. N. Nikolaev and V. I. Zakharov, Phys. Lett. B55 (1975) 397;

V. I. Zakharov and N.N.Nikolaev, Sov. J. Nucl. Phys. 21 (1975) 227;

A. H. Mueller and J. Qiu, Nucl. Phys. B268 (1986) 427;

E. L. Berger and J. Qiu, Phys. Lett. B206 (1988) 141;

F. E. Close and R. G. Roberts, Phys. Lett. B213 (1988) 91.

7) M. Glück and E. Reya, Phys. Lett. B79 (1978) 453;

E. L. Berger and D. Jones, Phys. Rev. D23 (1981) 1521;

R. Baier and R. Rückl, Z. Phys. C19 (1983) 251.

8) E. L. Feinberg and I. Ya. Pomeranchuk, Suppl. Nuovo Cim. III, Ser. X, No. 4 (1956) 652.

9) B. L. Ioffe, Phys. Lett. B130 (1969) 123.

10) M. L. Ter-Mikaelyan, J.E.T.P. 25 (1954) 289 and 296);

F. J. Dyson and H. Uberall, Phys. Rev. 99 (1955) 604.

11) V. N. Gribov, J.E.T.P. 30 (1970) 709.

12) See e.g.

A. Donnachie and G. Shaw, in Electromagnetic Interactions of Hadrons, A. Donnachie and G. Shaw (Eds.), Plenum Press, New York, 1978;

M. Arneodo, Preprint CERN-PPE/92-113, Phys. Rep., in press.

13) P. Amaudruz et al., Nucl. Phys. B371 (1992) 553.

14) J. Gomez et al., "Measurement of the A-Dependence of Deep-Inelastic Electron Scattering", Preprint SLAC-PUB-5813, August 1993.

15) S. Gupta and H. Satz, Z. Phys. C55 (1992) 391.

16) D. Kharzeev and H. Satz, Z. Phys. C60 (1993) 389.

17) D. M. Alde et al., Phys. Rev. Lett. 64 (1990) 2479. 


\section{Figure Captions:}

Figure 1:

Photoproduction of quarkonium on a nuclear target.

Figure 2:

A schematic view of shadowing and anti-shadowing regimes for photon and hadron interactions on a nuclear target, compared to a nucleon target, as function of the Bjorken variable $x_{t}$ of the target.

Figure 3:

Dilepton production via the basic Drell-Yan diagram (a), with hadronic fluctuations of the intermediate photon (b), and with higher order hadronic corrections to the $q \bar{q}$ annihilation process (c). 


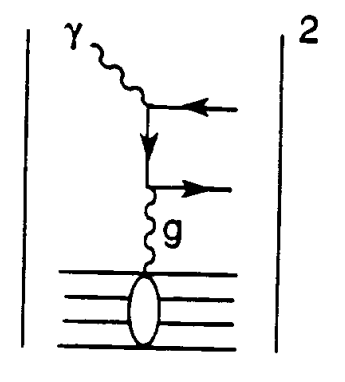

$=I \mathrm{~m}$

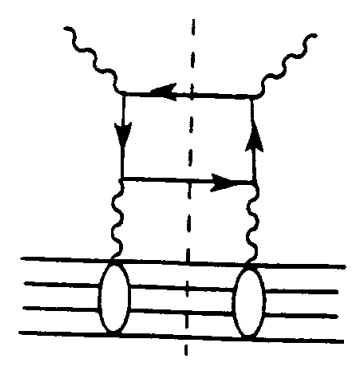

$=\mathrm{Im}$

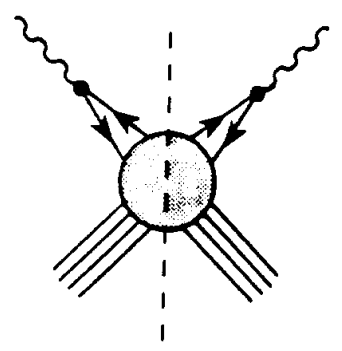

Fig. 1

Fig. 2

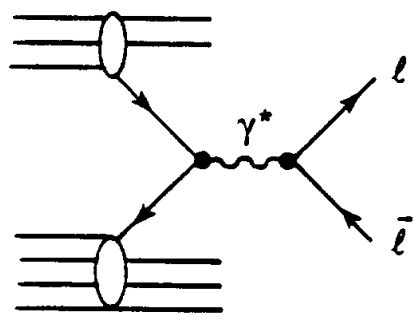

a)

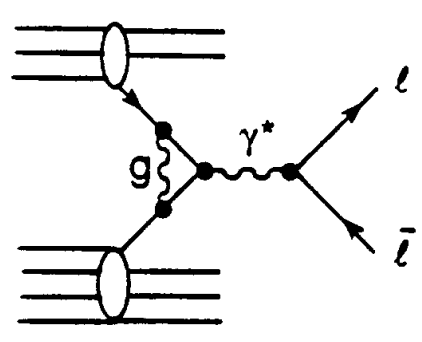

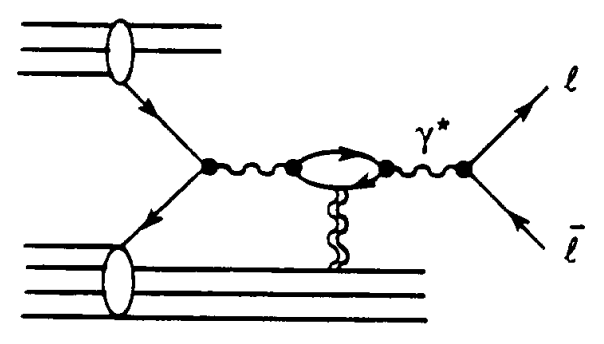

b)

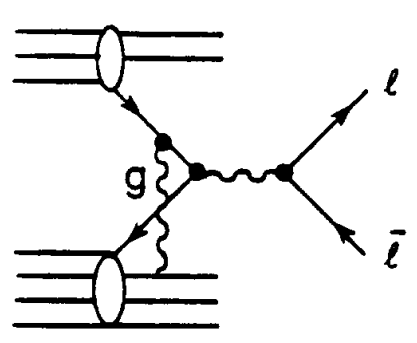

Fig. 3 
